\title{
The Origins of Early Sapiens Behaviour: Origins Centre, University of the Witwatersrand, Johannesburg
}

Yonatan Sahle

* Human Evolution Research Institute, Department of Archaeology, University of Cape Town, South Africa (₫yonatan.sahle@uct.ac.za)

This exhibition showcases the results of archaeological research at three coastal sites in the southern Cape of South Africa: Blombos Cave, Klipdrift Shelter and Klasies River main sites. Part of a long-term programme aiming to make palaeosciences accessible to the public, the exhibition befittingly started more locally, first at Stellenbosch and then at the Iziko South African Museums in Cape Town, before moving to Johannesburg. The exhibition opened at the Origins Centre of the University of the Witwatersrand on 25 November 2021. To those of us who attended the opening, it provided an opportunity to hear from the archaeologists, curators and designers behind the exhibition. The exhibition opened to the public on 27 November.

I visited the exhibition briefly on 25 November, and more thoroughly over the following days. The rich collections of unique artefacts undoubtedly offer visitors a fascinating glimpse into the minds of our human ancestors across 120-50 kya. Although not detailed in the exhibition, as recently as 25 years ago, behaviours that are recognised as quintessentially human were thought to have exclusively—and rather abruptly-emerged first in Eurasia, marking the onset of the so-called Upper Palaeolithic Revolution. By the close of the last century, however, unprecedented insights from genetic studies began to show that our species arose in Africa before spreading to the rest of the world. Fossil evidence for the oldest known Homo sapiens from Ethiopia soon augmented this emerging view. By this time, new dating techniques had already revealed that some of the suite of behaviours recognised as symbolic and complex appeared in Africa tens of thousands of years prior to the Upper Palaeolithic 'cultural explosion'. The early discoveries from Blombos Cave sit at the centre of this major shift in our understanding of how we began not only to look like but also to behave like humans. For informed visitors like myself, such historical backdrop of archaeological research makes the exhibition doubly appealing.

The select, rare artefacts on display dominate the exhibition and are supplemented by richly engaging media created by an Academy Award-winning documentary filmmaker. And the central theme of the exhibition - the evolution of uniquely human behaviours-resonates aptly with the meticulously upgraded spaces and permanent exhibits of the Origins Centre.

Visitors will find the ambience on the ground floor of the Origins Centre alluring. This is where the temporary exhibition starts, with a short video and textual description of how, as humans, "we are all one". It is also where one learns what may have facilitated the

(C) The Author(s), 2022. Published by Cambridge University Press on behalf of Antiquity Publications Ltd. This is an Open Access article, distributed under the terms of the Creative Commons Attribution licence (https://creativecommons. org/licenses/by/4.0/), which permits unrestricted re-use, distribution, and reproduction in any medium, provided the original work is properly cited. 


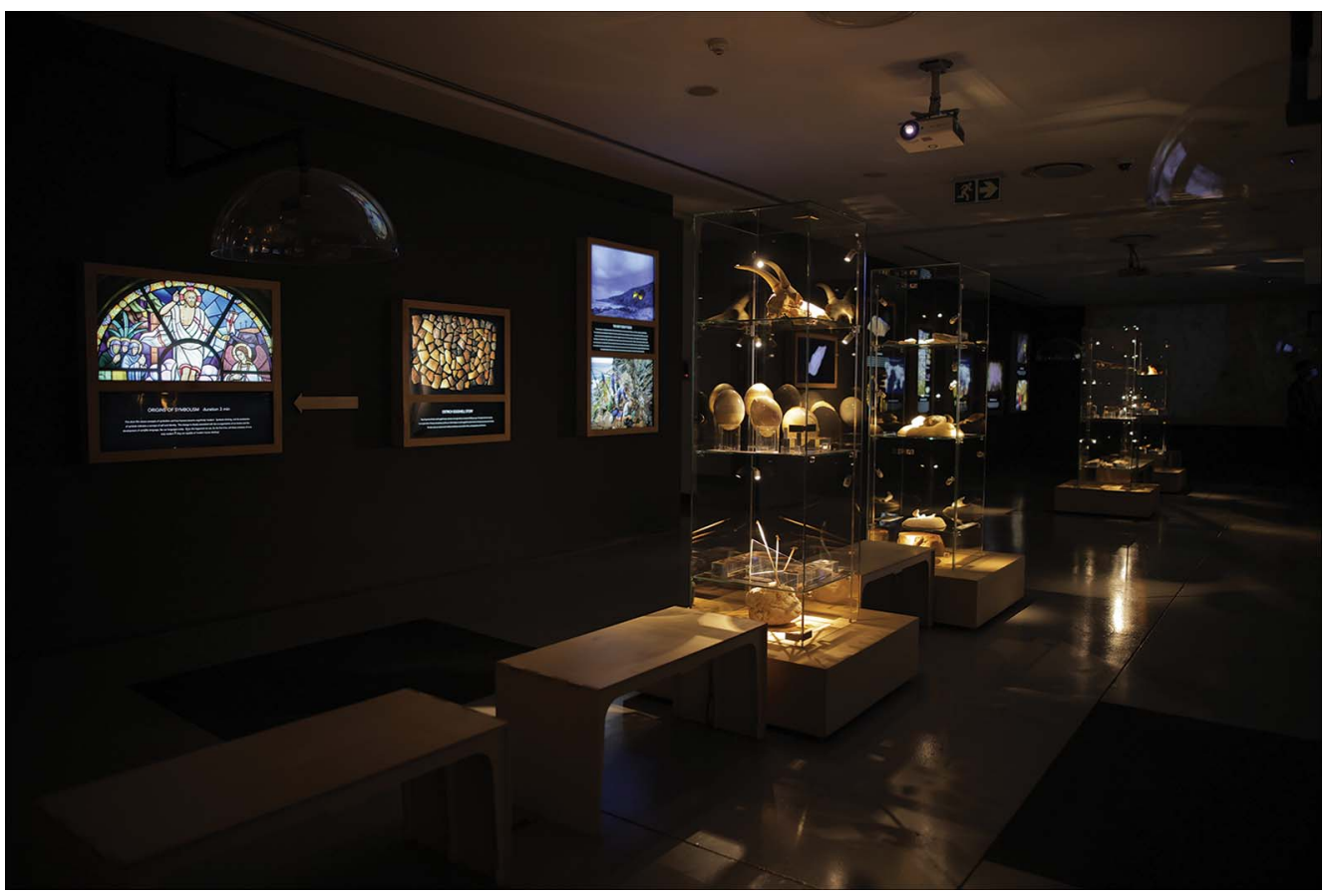

Figure 1. A partial view of the main exhibition (photograph courtesy of the Origins Centre, Witwatersrand).

development of such complex behaviours and technologies across the early Late Pleistocene landscapes of the southern Cape.

The main exhibition with displayed objects is installed in a room upstairs and contains around a dozen images beautifully illuminated by background lights (Figure 1). The first of these illustrates - with spectacular headshots of people - the fact that despite the seemingly immense diversity, we are all African in origin. Another image shows the Out-of-Africa dispersal of modern Homo sapiens after c. 100 kya. Beyond these, all other images are dedicated to showcasing exquisitely the iconic discoveries from the sites of interest: the remains of early Homo sapiens, complex projectile technologies and a variety of artefacts with symbolic implications. Evenly distributed between these are several screens with voice cones, each continuously replaying short (2-3-minute long) videos that explore different topics and sites and feature the main archaeologists behind the displayed discoveries.

The opposing, narrower walls of this rectangular gallery are dominated by two large screens depicting the evolution of complex technological innovation and behavioural adaptation among early humans, and, separately, an expanded view of the world's earliest ochre mixture and container. Finally, several tall glass cases, each with multiple compartments, display the actual artefacts and fossil remains from the three sites represented (Figure 2). Each display case is dedicated to specific types of finds, which are chronologically arranged and adequately captioned. The meaning and context of some of the iconic artefacts on display are represented by casts and exceptionally realistic replica models.

The exhibition lives up to its promise of making the science more engaging by incorporating an interactive visitor experience (Figure 3). A small room conveniently located at the

(C) The Author(s), 2022. Published by Cambridge University Press on behalf of Antiquity Publications Ltd. 


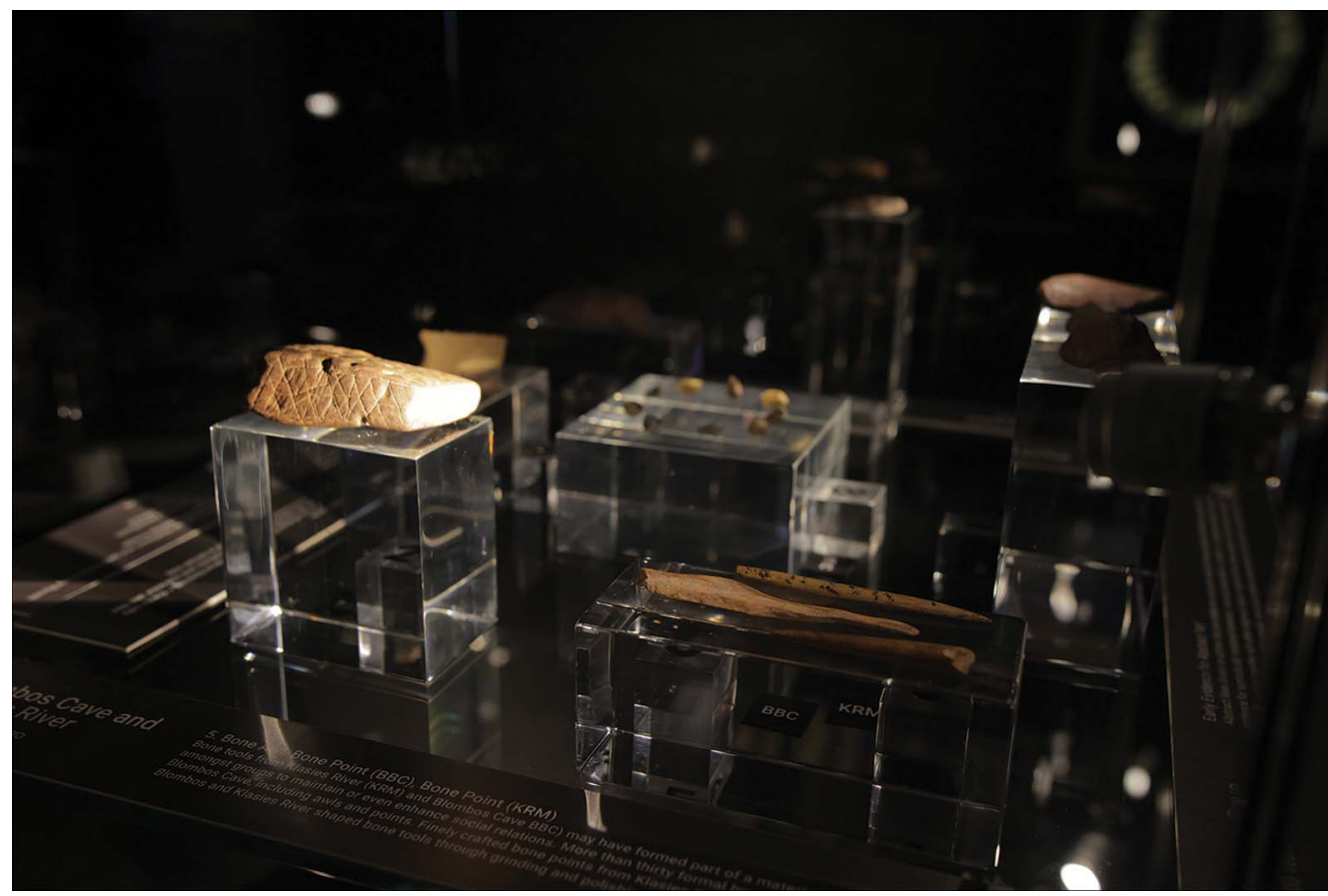

Figure 2. A close-up of the iconic engraved ochre slab from Blombos Cave, and pointed bone tools from Blombos Cave and Klasies River main sites (photograph courtesy of the Origins Centre, Witwatersrand).

end of the main exhibition is dedicated to a variety of richly engaging and educational activities, ranging from magnet boards for chronologically arranging the iconic finds from Blombos Cave, to ancient art puzzle pieces, an interactive light-box, and activity stations for making ochre paint and marking ostrich eggshells. This exceptional hands-on experience will certainly help visitors to better comprehend the details of early sapiens behaviour displayed in the main exhibition. For schoolchildren and visitors with youngsters, this room will undoubtedly be most suitable, enjoyable and impactful.

While the exhibition does cover the most important aspects of early sapiens behaviour via the rare finds from the three archaeological sites in the southern Cape and textual references to similar finds from other sites in this region, it is by no means without limitations. I found the exhibition title to be somewhat confusing, as the evidence on display does not in any way comprehensively capture the behavioural complexity of our early human ancestors documented outside of the southern Cape. In this regard, the visitor could be excused for expecting to see similarly remarkable discoveries from sites in other parts of the country, if not elsewhere on the continent. This is particularly reasonable for behaviours not documented in the southern Cape, such as mortuary practices and bedding construction. As such, there is now a growing opinion among mainstream researchers that the less extensively explored South African interior-and parts of North and eastern Africa-may have similarly been a crucible of such behaviours. If making this exhibition more comprehensive was too challenging, a clear delimitation of the exhibition's regional focus in its title would have sufficed.

(C) The Author(s), 2022. Published by Cambridge University Press on behalf of Antiquity Publications Ltd. 




Figure 3. A partial view of the interactive visitor experience: ostrich eggshell marking station (foreground) and ochre painting station (background) (photograph courtesy of the Origins Centre, Witwatersrand).

One may find the inclusion in the exhibition of a cast of the Kabwe cranium a little out of place, as most researchers consider it to represent a pre-sapiens lineage. Although similarly controversial, one wonders if the Florisbad cranium would be more appropriate here. Better yet, a cast of the earliest indisputably modern Homo sapiens cranium from Omo Kibish would have been ideal. Finally, one may find the main exhibition area to be a little too crowded in the approximately $6 \times 22 \mathrm{~m}$ main gallery. This is particularly apparent in the interference of the audio feeds from the various media devices, making it sometimes difficult to follow a specific source, even while standing under a voice cone.

Perhaps the strongest challenge for the exhibition will be attracting sufficient visitors amid rising cases of COVID-19 locally, including the Omicron variant just recently reported in the same province. It is to be hoped that COVID-19 restrictions will not prevent the exhibition's strong focus on, and appealing resources for, a tantalisingly interactive experience being fully appreciated by visitors. 